\title{
An Enhanced Local Binary Pattern for Texture Classification
}

\author{
Huawei Tao ${ }^{1}$, Rugang Wang ${ }^{1,2}$ and Li Zhao ${ }^{1 *}$ \\ ${ }^{1}$ School of Information Science and Engineering, Southeast University, Nanjing 210096, P.R.China \\ ${ }^{2}$ College of Information Engineering, Yancheng Institute of Technology, Yancheng, 224051, P.R. China \\ ${ }^{*}$ Corresponding author
}

\begin{abstract}
In order to improve the recognition rate of texture classification, an enhanced Local binary pattern, called completed local binary pattern based on gray level and structural information (CLBP_GLSI), is proposed in this paper. We firstly proposed an structural texture operator called gray level and structural information (GLSI), which adopts the average gray level of image to make image converted into binary images, and binary images are encoded as border or interior pixels image by Border/Interior Pixel Classification (BIC). Secondly, by combing with CLBP_M, CLBP_S and GLSI in into joint or hybrid distributions, the CLBP_GLSI are obtained. Experimental results obtained from two databases show that CLBP_GLSI achieves better results than other texture features.
\end{abstract}

Keywords-Completed Local binary Pattern (CLBP); Border/Interior Pixel Classification (BIC); Texture Classification

\section{INTRODUCTION}

Texture classification is a very important issue in computer vision and pattern recognition, what's more, it plays a very important role in many applications, such as face recognition, image retrieval et al [1-3]. Many methods about texture classification have been proposed during the past decades. Among them, the local binary pattern (LBP) proposed by Ojala is the most influential one [4].

Since Ojala's work, a large number of variants of LBP have been proposed. Tan et al.[5] proposed local Ternary pattern (LTP ) which quantizes the difference of two gray levels into three values. Subrahmanyam Murala [6] et al proposed a local tetra patterns which encodes the relationship among the center pixel and its neighbors according to the directions that are calculated using the first-order derivatives in vertical and horizontal directions. Guo et al.[7] proposed the completed local binary pattern (CLBP) which combines CLBP_M, CLBP_S, CLBP_C into joint or hybrid distribution and acquire better result. Khellah[8] proposed dominant neighborhood structure (DNS) which can acquire better result after combining with LBP. Motivated by CLBP, Zhao et al[9] proposed completed local count pattern (CLBC) and pointed out that micro-structure is not absolutely invariant to rotation, especially when the illumination conditions or the textural scales change a lot, but the recognition rate of CLBC is lower than that of CLBP in most texture databases. Song et al.[10] proposed noise-robust texture description by exploring a set of local contrast patterns via global measure for classification. Among above features, CLBP achieves better recognition results. However, CLBP_S and CLBP_M only consider the micro-structure information in texture images, while CLBP_C only represent gray level. Above three image descriptors all ignore the impact of macro-structure information in texture image, which may also important for texture classification.

Motivated by Border/Interior Pixel Classification [11], an new macro-structural texture operator, named gray level and structural information (GLSI) operator, is proposed. The GLSI not only contains the gray level information of image, but also contains macro-structure information of image. Similar as CLBP, GLSI is combined with CLBP_M, CLBP_S, then an new texture feature, called completed local binary pattern based on gray level and structural information, are proposed. Experimental results on two databases demonstrate that CLBP_GLSI achieves better results in two databases.

\section{RELATED THEORY}

\section{A. Completed Local Binary Pattern}

Guo et al [7] proposed CLBP by decomposing the image local difference into two complementary components: the signs and the magnitudes, and two operators named CLBP_S and CLBP_M. CLBP_S and CLBP_M can be defined as:

$$
\begin{aligned}
& C L B P_{-} S_{P, R}=\sum_{p=0}^{P-1} s\left(g_{p}-g_{c}\right) 2^{P} s(x)=\left\{\begin{array}{l}
1, x \geq 0 \\
0, x<0
\end{array}\right. \\
& C L B P_{-} M_{P, R}=\sum_{p=0}^{P-1} t\left(m_{p}, c\right) 2^{P} m_{p}=\left|g_{p}-g_{c}\right|
\end{aligned}
$$

where $g_{c}$ is the gray value of the center pixel and $g_{p}$ is the gray value of the neighbor pixel on a circle of radius $R$, and $P$ is the total number of the neighbors, $C$ is the mean of $m_{p}$ of the whole image, $t(x, c)$ are defined as in Eqn. (3).

$$
t(x, c)=\left\{\begin{array}{l}
1, x \geq c \\
0, x<c
\end{array}\right.
$$


As the image local gray level also has discriminative information, an operator called CLBP_center (CLBP_C) is proposed, which represents the image gray level. CLBP_C is defined as follow:

$$
C L B P C_{P, R}=t\left(g_{c}, C_{l}\right)
$$

where $C_{l}$ is the average gray value of the whole image gray value. $g_{c}$ is the value of the central pixel.

\section{PROPOSED METHOD}

\section{A. Gray Level and Structural Information operator}

In order to fuse macro-structure into texture operator, a new operator called Gray Level and Structural Information operator (GLSI) is proposed. The encode steps are as follow:

(1) The original image is processed by CLBP_C encoding and CLBP_C binary image is obtained.

(2) After the 1 step, CLBP_C binary image pixels are classified as border or interior pixels. As formula (5) show, a pixel is classified as interior if its 4-neighbors have the same pixel value. And a pixel is classified as border if it is at the border of the image itself or if at least one of its 4-neighbors has the different pixel value. After above processing, a binary called BIC binary image is obtained.

$$
\left\{\begin{array}{l|l}
\text { if } f(i, j) \text { is interior } & \begin{array}{l}
f(i, j)=f(i+1, j) \\
f(i, j)=f(i-1, j) \\
f(i, j)=f(i, j+1) \\
f(i, j)=f(i, j-1)
\end{array}
\end{array}\right\}
$$

(3) After the 2 step, all the pixels of BIC binary image plus 1 , if a pixel is an interior, the pixel multiply by -1 , or it remains unchanged.

After the above three step, we can get the GLSI image which not only represent the image gray level information, but also fuse the macro-structure feature of the image. In FIGURE I (a), we can see that the traditional CLBP_C only capture gray level information; in the FIGURE I(b), the CLBP_C image has been classified as two class, and the BIC image contain gray level information and structural information. GLSI will be used to combine with other local texture operator, so we process the BIC image as step (3) did. After step (3), the GLSI becomes a 4 value image which contains gray level information and structure information.

\section{B. Completed Local Binary Pattern Based on Gray Level and Structural Information}

Similar as CLBP, a new texture feature called completed local binary pattern based on gray level and structural information is proposed, which contain three operators: CLBP_S, CLBP_M, GLSI, where CLBP_S is described in formula (1), CLBP_M is described in formula (2). GLSI is described in section $\mathrm{A}$

Similar as [9], two means are adopted to combine different operators: jointly and hybridly, respectively. As FIGURE IIshows, in the jointly means, a 2-D joint histogram, "CLBP_M/GLSI", is built, and then the histogram is converted to a 1-D histogram, which is concatenated with CLBP_M to generate a joint histogram, denoted by "CLBP_S_M / GLSI". In the hybrid means, a 3-D joint histogram is built, and then the histogram is converted to a 1-D histogram, denoted by "CLBP S/M/GLSI”.

\begin{tabular}{|l|l|l|l|l|l|l|l|}
\hline 1 & 1 & 1 & 0 & 1 & 0 & 1 & 0 \\
\hline 1 & 1 & 1 & 1 & 0 & 0 & 0 & 0 \\
\hline 1 & 1 & 1 & 1 & 0 & 0 & 1 & 1 \\
\hline 1 & 0 & 1 & 0 & 0 & 0 & 1 & 1 \\
\hline 0 & 1 & 1 & 0 & 0 & 1 & 0 & 0 \\
\hline 1 & 1 & 1 & 1 & 1 & 0 & 0 & 0 \\
\hline 1 & 1 & 1 & 1 & 1 & 1 & 0 & 1 \\
\hline 0 & 1 & 1 & 1 & 1 & 0 & 1 & 0 \\
\hline
\end{tabular}

(a) CLBP_C binary image

\begin{tabular}{|l|l|l|l|l|l|l|l|}
\hline 1 & 1 & 1 & 0 & 1 & 0 & 1 & 0 \\
\hline 1 & 1 & 1 & 1 & 0 & 0 & 0 & 0 \\
\hline 1 & 1 & 1 & 1 & 0 & 0 & 1 & 1 \\
\hline 1 & 0 & 1 & 0 & 0 & 0 & 1 & 1 \\
\hline 0 & 1 & 1 & 0 & 0 & 1 & 0 & 0 \\
\hline 1 & 1 & 1 & 1 & 1 & 0 & 0 & 0 \\
\hline 1 & 1 & 1 & 1 & 1 & 1 & 0 & 1 \\
\hline 0 & 1 & 1 & 1 & 1 & 0 & 1 & 0 \\
\hline
\end{tabular}

(b) BIC binary image

\begin{tabular}{|c|c|c|c|c|c|c|c|}
\hline 2 & 2 & 2 & 1 & 2 & 1 & 2 & 1 \\
\hline 2 & -2 & -2 & 2 & 1 & -1 & 1 & 1 \\
\hline 2 & 2 & -2 & 2 & 1 & 1 & 2 & 2 \\
\hline 2 & 1 & 2 & 1 & -1 & 1 & 2 & 2 \\
\hline 1 & 2 & 2 & 1 & 1 & 2 & 1 & 1 \\
\hline 2 & -2 & -2 & 2 & 2 & 1 & -1 & 1 \\
\hline 2 & -2 & -2 & -2 & -2 & 2 & 1 & 2 \\
\hline 1 & 2 & 2 & 2 & 2 & 1 & 2 & 1 \\
\hline
\end{tabular}

FIGURE I. THE ILLUSTRATION OF THE GLSI PROCESSING STEPS 


\section{SIMULATIONS}

\section{A. Evaluation measure}

In order to evaluate the performance of algorithms, the $\chi^{2}$ statistics is used as the dissimilarity between two histograms [7,9]. The $\chi^{2}$ statistics can be calculated as follows:

$$
d_{\chi^{2}}(H, K)=\sum_{i=1}^{B} \frac{\left(h_{i}-k_{i}\right)^{2}}{h_{i}+k_{i}}
$$

where $h_{i}$ and $k_{i}$ are the elements of histograms $H$ and $K$.

In this paper, the nearest neighborhood classifier is used for classification.

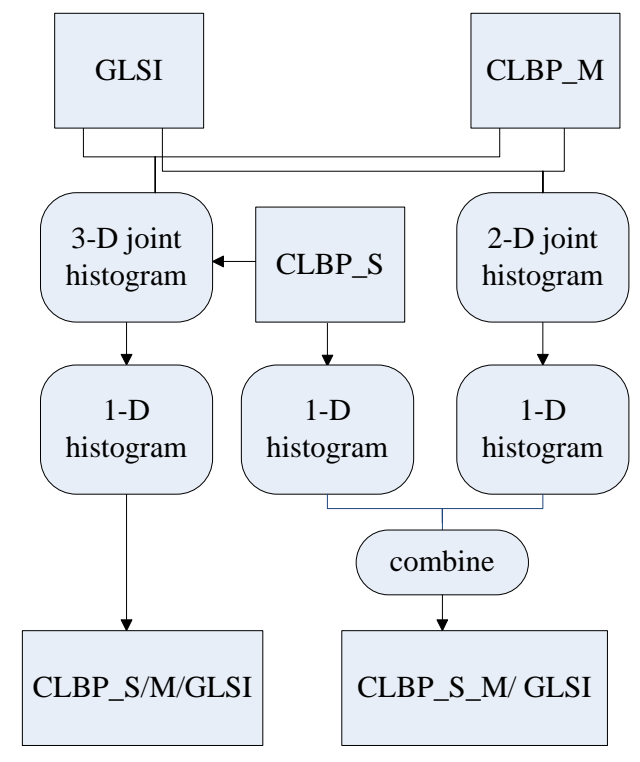

FIGURE II. JOINTLY AND HYBRIDLY MEANS.

\section{B. Experimental results on Outex database}

A series of experiments are carried out on the Outex database. For the Outex [12], we use Outex_TC_00010(TC10) and Outex_TC_00012 (TC12) as the simulation database. There are the same 24 classes of texture in TC10 and TC12, where each class with 20 images $(128 \times 128$ pixels $)$ is designed for rotational invariant texture classification. And there are contains three different simulated illuminations ("inca","horizon" and "tl84") for each class, where each illumination includes nine different rotation angles $\left(0^{\circ}, 5^{\circ}, 10^{\circ}\right.$, $15^{\circ}, 30^{\circ}, 45^{\circ}, 60^{\circ}, 75^{\circ}$ and $90^{\circ}$ ).

Table I shows the experimental results. Some interesting finding can be found from Table I. (1) In jointly means, CLBP_S_M/GLSI achieves better result than CLBP_S_M/C and CLBC_S_M/C. Compared with CLBP_S_M/C, the accurate rate (AR) of CLBP_S_M/GLSI are averagely improved by $5.06 \%, 1.58 \%, 1.11 \%$ when $\mathrm{R}=1, \mathrm{R}=2$ and $\mathrm{R}=3$.
This means GLSI provides more structural information for CLBP_S_M/GLSI. (2) In hybridly means, The CLBP_S/M/GLSI also achieves better results than other features. Compared with CLBP_S/M/C, the AR of CLBP_S/M/GLSI are averagely improved by $1.51 \%, 0.37 \%$, $0.16 \%$ when $\mathrm{R}=1, \mathrm{R}=2$ and $\mathrm{R}=3$. (3) The dimensions of CLBP_S_M/ GLSI are 45, 85, 125 when $\mathrm{R}=1, \mathrm{R}=2$ and $\mathrm{R}=3$, and the dimensions of CLBP_S/M/C are 200, 648, 1352. It is worth noting that the AR of CLBP_S_M/ GLSI gets close to that of CLBP_S/M/C. Especially when $\mathrm{R}=3$, the average AR of CLBP_S_M/GLSI is $96.04 \%$, while the average AR of CLBP_S/M/C is $96.26 \%$, the former's dimensions are far lower than the latter's dimensions. (4) It can be seen from TABLE I, the best AR in all cases are achieved by CLBP_S/M/GLSI and CLBP_S_M/GLSI. Based on above finding, GLSI extract more information from texture images, which make CLBP_GLSI achieved better experimental results.

\section{Experimental Results on CURet Database}

The CURet database [13] includes 61 kinds of texture image, there are 205 images obtained from different viewpoint and illumination orientations for each kind of texture image. 92 images whose polar angle less than 0.909333 are chose in this paper for simulation, where a sufficiently large region could be cropped $(128 * 128)$ across all texture classes. all the cropped regions are converted into grey scale. As in [4], $\mathrm{N}$ images were randomly chosen as training samples from each class, and the remaining $(92-\mathrm{N})$ were used as test samples. The average classifications accrete rates for a hundred random splits are listed in TABLEII.

From TABLE II, we can get several finding like Table I. (1) In jointly means, CLBP_S_M/GLSI also achieve best AR compare to that of CLBP_S_M/C and CLBC_S_M/C. The best results for CLBP_S_M/GLSI are 95.21\%, 84.58\%, 74.68\% for 46,12,6, while the best results for CLBP_S_M/C are 93.64\%, $82.44 \%, 72.28 \%$. The AR of the former are improved by $1.57 \%$, 2.14\%, 2.4\%. (2) In hybridly means, CLBP_S/M/GLSI still obtains the better AR than that of CLBP_S/M/C and CLBC_S/M/C. The best results for CLBP_S/M/GLSI are 95.95\%, 86.39\%, 76.95\% for 46,12,6, while the best results for CLBP_S/M/C are $95.47 \%, 85.51 \%, 75.83 \%$. The AR of the former are improved by $0.48 \%, 0.88 \%, 1.12 \%$. (3) Among all of feature, CLBP_S/M/GLSI achieves the best AR.

The experimental results on Outex and CURet show that CLBP_GLSI achieves better results than other features. Compared with CLBP, CLBP_GLSI with GLSI achieve better results, which means that GLSI provides more information for texture feature. In addition, the AR of CLBP_S_M/GLSI gets close to that of CLBP_S/M/C and CLBP_S/M/GLSI in both databases, while the dimensions of the former are far lower than that the latter. 
TABLE I. EXPERIMENTAL RESULTS IN OUTEX DATABASE

\begin{tabular}{|c|c|c|c|c|c|c|c|c|c|c|c|c|}
\hline & \multicolumn{4}{|c|}{$R=1, P=8$} & \multicolumn{4}{|c|}{$\mathrm{R}=2, \mathrm{P}=16$} & \multicolumn{4}{|c|}{$R=3, P=24$} \\
\hline & \multirow[t]{2}{*}{ TC10 } & \multicolumn{2}{|c|}{ TC12 } & \multirow[t]{2}{*}{ Average } & \multirow[t]{2}{*}{ TC10 } & \multicolumn{2}{|c|}{ TC12 } & \multirow[t]{2}{*}{ Average } & \multirow[t]{2}{*}{ TC10 } & \multicolumn{2}{|c|}{ TC12 } & \multirow[t]{2}{*}{ Average } \\
\hline & & T184 & horizon & & & T184 & horizon & & & T184 & horizon & \\
\hline LTP & 94.14 & 75.88 & 73.96 & 81.33 & 96.95 & 90.16 & 86.94 & 91.35 & 98.20 & 93.59 & 89.42 & 93.74 \\
\hline CLBP_S_M/C & 94.53 & 81.87 & 82.52 & 86.31 & 8.02 & 90.99 & 91.08 & 93.36 & 8.33 & 94.05 & 92.40 & 94.93 \\
\hline CLBC_S_M/C & 93.75 & 81.85 & 83.40 & 86.33 & 96.93 & 89.63 & 90.23 & 92.26 & 97.21 & 88.77 & 89.61 & 91.86 \\
\hline $\begin{array}{l}\text { CLBP_S_M/ } \\
\text { GLSI }\end{array}$ & 96.41 & 87.87 & 89.84 & 91.37 & 98.65 & 93.17 & 93.01 & 94.94 & 98.93 & 95.16 & 94.03 & 96.04 \\
\hline CLBC_S/M/C & 97.16 & 89.79 & 92.92 & 93.29 & 98.54 & 93.26 & 94.07 & 95.29 & 98.78 & 94.00 & 93.24 & 95.67 \\
\hline CLBP_S/M/C & 96.56 & 90.30 & 92.29 & 93.05 & 98.72 & 93.54 & 93.91 & 95.39 & 98.93 & 95.32 & 94.53 & 96.26 \\
\hline CLBP_S/M/GLSI & 97.64 & 91.78 & 94.26 & 94.56 & 98.85 & 94.03 & 94.40 & 95.76 & 98.83 & 95.88 & 94.54 & 96.42 \\
\hline
\end{tabular}

TABLE II. EXPERIMENTAL RESULTS ON CURET DATABASE

\begin{tabular}{cccccccccc}
\hline & \multicolumn{3}{c}{$\mathbf{R}=\mathbf{1} \mathbf{P}=\mathbf{8}$} & \multicolumn{3}{c}{$\mathbf{R}=\mathbf{2 ,} \mathbf{P}=\mathbf{1 6}$} & \multicolumn{3}{c}{$\mathbf{R}=\mathbf{3} \mathbf{P}=\mathbf{2 4}$} \\
The value of N & $\mathbf{4 6}$ & $\mathbf{1 2}$ & $\mathbf{6}$ & $\mathbf{4 6}$ & $\mathbf{1 2}$ & $\mathbf{6}$ & $\mathbf{4 6}$ & $\mathbf{1 2}$ & $\mathbf{6}$ \\
\hline LTP & 86.49 & 72.26 & 61.61 & 90.53 & 77.92 & 67.51 & 91.58 & 79.76 & 69.77 \\
CLBP_S_M/C & 90.11 & 77.03 & 66.19 & 92.17 & 80.29 & 69.63 & 93.64 & 82.44 & 72.28 \\
CLBC_S_M/C & 88.09 & 73.89 & 62.98 & 89.69 & 76.05 & 64.93 & 90.12 & 76.50 & 65.55 \\
CLBP_S_M/GLSI & 94.26 & 82.50 & 72.02 & 94.97 & 83.97 & 73.87 & 95.21 & 84.58 & 74.68 \\
CLBP_S/M/C & 95.47 & 85.17 & 74.83 & 95.32 & 85.45 & 75.42 & 95.23 & 85.51 & 75.83 \\
CLBC_S/M/C & 94.82 & 83.27 & 72.37 & 94.71 & 83.66 & 72.76 & 93.57 & 81.94 & 71.00 \\
CLBP_S/M/GLSI & $\mathbf{9 5 . 9 5}$ & $\mathbf{8 6 . 2 8}$ & $\mathbf{7 6 . 9 5}$ & $\mathbf{9 5 . 7 4}$ & $\mathbf{8 6 . 3 9}$ & $\mathbf{7 6 . 6 7}$ & $\mathbf{9 5 . 5 1}$ & $\mathbf{8 6 . 0 5}$ & $\mathbf{7 6 . 3 9}$ \\
\hline
\end{tabular}

\section{CONCLUSIONS}

In this paper, a texture feature, called completed local binary pattern based on gray level and structural information is proposed. Firstly, structural texture operator called gray level and structural information (GLSI) is proposed, Secondly, by combing with CLBP_M, CLBP_S and GLSI in into joint or hybrid distributions, the CLBP_GLSI are obtained. Experimental results show that CLBP_GLSI achieve best results, compared to other texture features, in joint or hybrid means. In addition, the AR of CLBP_GLSI in joint means get close to CLBP_S/M/C and CLBP_S/M/GLSI in hybrid means, while the dimensions of the former are far lower than the latter.

\section{ACKNOWLEDGMENT}

The work was supported by the National Natural Science Foundation of China under Grant No. 61673108, 61571106 and 61375028. China Postdoctoral Science Foundation funded project under Grant No. 2016M601695. The authors also gratefully acknowledge the helpful comments and suggestions of the reviewers, which have improved the presentation.

\section{REFERENCES}

[1] Xi Chen, Zaihong Zhou, Jiashu Zhang, et al. "Local convex-and-concave pattern :an effective texture descriptor,” Information Sciences, vol.236, pp.120-139,2016

[2] Shiv Ram Dubey, Satish Kumar Singh, Rajat Kumar Singh. "Multichannel Decoded Local Binary Patterns for Content Based Image Retrieval,” IEEE Trans. Image Processing, vol. 25, no. 9, pp.4018-4032, July 2016.

[3] Bruno Dot, Do nascimento MZ, Ramos RP, et al. "LBP operators on curvelet coefficients as an algorithm to describe texture in breast cancer tissues," vol. 55, pp. 329-340, August 2015.

[4] T. Ojala, M. Pietikäinen,T Mäenpää, ”Multiresolution Gray-Scale and Rotation Invariant Texture Classification with Local Binary Patterns," IEEE Trans. On Pattern Analysis and Machine Intelligence, vol.24, no.7, pp.971-987, July 2002.
[5] X. Tan and B. Triggs, "Enhanced local texture feature sets for face recognition under difficult lighting conditions," IEEE trans. Image Process., vol.19, no.6,pp.1635-1650, June 2010.

[6] Subrahmanyam Murala, R. P. Maheshwari, R. Balasubramanian, "Local Tetra Patterns: A New Feature Descriptor for Content-Based Image Retrieval,” IEEE trans. Image Process., Vol. 21, No. 5, pp. 2874-2885, may 2012.

[7] Z.Guo, L.Zhang, and D. Zhang, "A completed modeling of local binary pattern operator for texture classification,” IEEE trans. Image Process., vol.19, no.6, pp.1657-1663, June 2010.

[8] F.Khellah, "Texture classification using dominant neighborhood structure,” IEEE trans. Image Process.,vol.20, no.11,pp.3270-3279, Nov. 2011.

[9] Y.Zhao, D.Huang, and W. Jia, "Completed Local Binary Count for Rotation Invariant Texture Classification,” IEEE trans. Image Process., vol. 21, no.10, pp.4492-4497, Oct. 2012.

[10] T.Song, H.LI, and F.Meng, et.al, "Noise-Robust Texture Description Using Local Contrast Pattern via Global Measures,” IEEE signal processing letter, vol.21, no.1, pp.93-96, January 2014.

[11] R. Stehling, M. A. Nascimento, A. X. Falcão. ”A compact and efficient image retrieval approach based on border/interior pixel classification", Proceedings of the eleventh international conference on Information and knowledge management, New York, USA, pp.102-109, 2002.

[12] T. Ojala, T. Maenpaa, M. Pietikainen, J. Viertola, J. Kyllönen, and S.Huovinen, "Outex-new framework for empirical evaluation of texture analysis algorithms,” in Proc. 16th Int. Conf. Pattern Recognit., vol. 1. pp. 701-706, 2002. 